PTPN 14

functions

within the p53

pathway

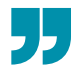

\title{
Digging deeper into p53's functions
}

Although the tumour suppressor p53 (encoded by Trp53 in mice and TP53 in humans) has been extensively studied for many years, the mechanisms by which $\mathrm{p} 53$ prevents tumour formation are still unclear. Mello et al. analysed the effects of various p53 transactivation domain (TAD) mutants in pancreatic ductal adenocarcinoma (PDAC) and uncovered a crucial tumour-suppressive pathway in which p53 mediates inhibition of the transcriptional co-activator YAP.

Using mice that express oncogenic $\mathrm{Kras}^{\mathrm{G} 12 \mathrm{D}}$ in the pancreas, the authors showed that expression of a $\operatorname{Tr} p 53$ allele with both TADs mutated functions as a Trp53-null allele and promotes PDAC development. By contrast, mice that express Kras $^{\mathrm{G} 12 \mathrm{D}}$ and a $\operatorname{Trp} 53$ allele with only the second TAD mutated $\left(\operatorname{Tr} p 53^{53,54}\right)$ are protected from PDAC development, suggesting that $\operatorname{Tr} p 53^{53,54}$ functions as a 'super' tumour suppressor. Transcriptomic and chromatin immunoprecipitation followed by sequencing (ChIP-seq) data indicated that $\operatorname{Tr} p 53^{53,54}$ hyperactivates target genes in mouse embryonic fibroblasts (MEFs).

Of the genes with potential relevance in PDAC, the authors selected protein tyrosine phosphatase non-receptor type 14

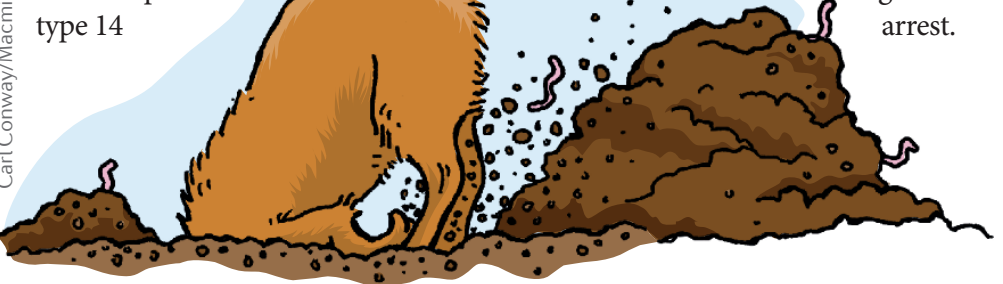

(Ptpn14) for further study. PTPN14 directly binds to and negatively regulates YAP, a protein that promotes PDAC progression. ChIP-seq data indicated that p53 binds to the PTPN14 locus in primary human fibroblasts and MEFs, and PTPN14 expression was p53-dependent in a variety of different oncogene-expressing mouse and human cells. In addition, PTPN14 expression was hyperactivated by $\operatorname{Trp} 53^{53,54}$.

In Kras ${ }^{\mathrm{G} 12 \mathrm{D}} ; \operatorname{Tr} p 53^{-/-}$mouse PDAC cells and in TP53-mutant human PDAC cells, PTPN14 overexpression inhibited proliferation, clonogenic potential and anchorage-independent growth. Knockdown of PTPN14 in p53-expressing PDAC cells promoted clonogenic and anchorage-independent growth in vitro and subcutaneous growth in mice, whereas knockdown of PTPN14 in p53-deficient PDAC cells had no effect, indicating that PTPN14 functions within the $\mathrm{p} 53$ pathway. Importantly, knockdown of PTPN14

or p53 had similar effects, suggesting a crucial role for PTPN14 in this tumour-suppressive pathway.

Mutants of PTPN14 that could not bind to YAP were unable to mediate PDAC cell DAC cell
growth growth ORIGINAL ARTICLE Mello, S. S. et al. A p53 supertumor suppressor reveals a tumor suppressive p53-Ptpn14-Yap axis in pancreatic cancer. Cancer Cell 32, 460-473 (2017)

FURTHER READING Bieging, K. T., Mello, S. S. \& Attardi, L. D. Unravelling mechanisms of p53-mediated tumour suppression. Nat. Rev. Cancer 14, 359-370 (2014) 